\title{
Baseline Values of Candidate Urine Acute Kidney Injury Biomarkers Vary by Gestational Age in Premature Infants
}

\author{
DAVID J. ASKENAZI, RAJESH KORALKAR, EMILY B. LEVITAN, STUART L. GOLDSTEIN, PRASAD DEVARAJAN, \\ SRIKRISHNA KHANDRIKA, RAVINDRA L. MEHTA, AND NAMASIVAYAM AMBALAVANAN
}

\begin{abstract}
Department of Pediatrics [D.J.A., N.A.], School of Public Health [R.K., E.B.L.], University of Alabama at Birmingham, Birmingham, Alabama 35233; Department of Pediatrics [S.L.G., P.D.], Cincinnati Children's Hospital and Medical Center, Cincinnati, Ohio 45229; Department of Medicine [R.L.M., S.K.], University of California at San Diego, San Diego, California 92103
\end{abstract}

\begin{abstract}
Acute kidney injury (AKI) is common in premature infants and is associated with poor outcomes. Novel biomarkers can detect AKI promptly. Because premature infants are born with underdeveloped kidneys, baseline biomarker values may differ. We describe baseline values of urinary neutrophil gelatinase-associated lipocalin (NGAL), IL-18, kidney injury molecule-1 (KIM-1), osteopontin (OPN), beta-2 microglobulin (B2mG), and Cystatin-C (CysC). Next, we test the hypothesis that these biomarkers are inversely related to GA. Candidate markers were compared according to GA categories in 123 infants. Mixed linear regression models were performed to determine the independent association between demographics/interventions and baseline biomarker values. We found that urine NGAL, KIM-1, Cys-C, and B2mG decreased with increasing GA. With correction for urine creatinine (cr), these markers and OPN/cr decreased with increasing GA. IL-18 (with or without correction for urine creatinine) did not differ across GA categories. Controlling for other potential clinical and demographic confounders with regression analysis shows that NGAL/cr, OPN/cr, and B2mG/cr are independently associated with GA. We conclude that urine values of candidate AKI biomarkers are higher in the most premature infants. These findings should be considered when designing and analyzing biomarker studies in newborn with AKI. (Pediatr Res 70: 302-306, 2011)
\end{abstract}

A lthough outcomes in very LBW (VLBW) premature infants have improved over the past few decades, morbidity and mortality continue to be high (1). Acute kidney injury (AKI) is common and may be independently associated with mortality (2-4) in VLBW infants. Our ability to improve outcomes in those with AKI is hampered by the inability to detect AKI early in the disease process. Novel urine biomarkers of AKI have been discovered and promise to reliably detect AKI before an increase in serum creatinine $(\mathrm{SCr})$ in different critically ill populations (5-14). Many of the biomarkers available have been tested in newborns undergoing cardiopulmonary bypass $(15,16)$ but must be evaluated in VLBW infants who are born with underdeveloped glomeruli and tubules.

Our current methods to diagnose AKI using SCr-based definitions are problematic for the following reasons (17-19):

Received July 30, 2010; accepted March 28, 2011.

Correspondence: David J. Askenazi, M.D., Division of Nephrology, Department of Pediatrics, University of Alabama at Birmingham, 1600 7th Ave South, ACC 516, Birmingham, AL 35233; e-mail: daskenazi@peds.uab.edu

Supported by a pilot and feasibility grant from the NIH O'Brien Center for Acute Kidney Injury, the National Kidney Foundation Young Investigator's Award, and the Kaul Pediatric Research Institute grant. a) changes in $\mathrm{SCr}$ represent a functional abnormality that occurs as a consequence of injury, not a marker of injury; b) SCr concentrations may not change until $25-50 \%$ of the kidney function has already been lost and thus it may take days before a significant increase in $\mathrm{SCr}$ is seen; c) $\mathrm{SCr}$ varies by muscle mass, hydration status, age and gender; d) $\mathrm{SCr}$ reflects maternal levels at birth and normally decreases to represent the infant's kidney function in the first few weeks of life (depending on the level of prematurity); and finally, e) blood analysis is not without consequences in premature infants as their total blood volume can be quite low (estimated blood volume of $500 \mathrm{~g}$ infant is $40 \mathrm{~mL}$ ).

Recent advances in the field of clinical proteomics have greatly accelerated the discovery of novel urinary proteins which promptly increases in response to renal tubular injury. Some of the most promising urine AKI biomarkers include neutrophil gelatinase-associated lipocalin (NGAL), (20), IL-18 (16), kidney injury molecule-1 (KIM-1) (21,22), osteopontin (OPN), beta-2 microglobulin (B2mG), and Cystatin-C (Cys-C) (23). These and other biomarkers are being tested extensively in different critically ill populations, including children $(3,6,14,18,24,25)$ and hold the promise to change our approach to AKI as they can detect AKI hours after an insult as opposed to $\mathrm{SCr}$ which may take days to increase after an injury. Development of these biomarkers has advanced such that point-of-care biomarker assessment kits are now becoming available for serum and urine NGAL $(7,19)$ and KIM-1 (26).

Because glomerular and tubular development continues until 34 wk after GA, baseline levels of candidate biomarkers may be different depending on the degree of prematurity. Baseline evaluation of urine NGAL in premature infants has been performed and shows that urine biomarkers are inversely related to both GA and birth weight (27). Confirmation of these findings and exploration of the effect that patient demographics and clinical interventions have on other candidate biomarkers are needed. To determine the baseline levels of

\footnotetext{
Abbreviations: AKI, acute kidney injury; AKIN, Acute Kidney Injury Network; B2mG, beta-2 microglobulin; cr, creatinine; Cys-C, Cystatin-C; 2-plex, Duplex; 4-plex, four value multiplex; KIM-1, kidney injury molecule-1; NGAL, neutrophil gelatinase-associated lipocalin; MSD, Meso Scale Discovery; OPN, osteopontin; SCr, serum creatinine; TPA, tripropylamine; VLBW, very VLBW
} 
candidate AKI biomarkers in premature infants, we collected urine from premature infants during the first 7 postnatal days to test the hypothesis that urine levels of NGAL, KIM-1, IL-18, OPN, B2mG, and Cys-C are dependent on the degree of prematurity.

\section{METHODS}

VLBW infants with birth weight between 500 and $1500 \mathrm{~g}$ admitted to the regional quaternary care NICU at the University of Alabama at Birmingham (UAB) between February 2008 and July 2009 were enrolled in the study. Parental consent was obtained, and the study was approved by the Institutional Review Board at UAB. Infants were excluded if they did not survive to $48 \mathrm{~h}$ of life or if they had known congenital abnormality of the kidney.

Demographics. Infant demographic data obtained consisted of GA, birth weight, race, small for GA, and sex. Clinical data included administration of aminoglycoside antibiotics and/or indomethacin, ventilator support, and Apgar scores at 1 and $5 \mathrm{~min}$. Maternal demographic data collected included age, diabetes, pregnancy-induced hypertension, and preeclampsia. Maternal clinical data included administration of corticosteroids, aminoglycosides, vancomycin, and indomethacin.

Definition of AKI was made if an infant met criteria for Stage 1 definition (an increase in SCr by $>0.3 \mathrm{mg} / \mathrm{dL}$ in $48 \mathrm{~h}$ or an increase of $>50 \%$ from a previous value) of the AKIN (Acute Kidney Injury Network) classification, within the first 7 postnatal days (28). SCr was analyzed from laboratory results obtained as part of routine care, and if available from remnant samples that would have otherwise been discarded.

Biomarker analysis. Urine was collected during the first 7 postnatal days with cotton balls placed near the perineum. Urine was extracted and centrifuged for $10 \mathrm{~min}$ to remove any cotton fibers or cellular elements and then frozen at $-70^{\circ} \mathrm{C}$ until sample evaluation. Urine biomarker analysis was performed by Core A of the National Institutes of Health P30 O'Brien Core Center for AKI research (www.obrienaki.org) using the Meso Scale Discovery (MSD; Gaithersburg, MD).

NGAL, Cys-C, OPN, and B2mG were measured in urine with a prototype four value multiplex (4-plex) assay. IL-18 and KIM-1 were measured with a prototype duplex (2-plex) assay developed for this study.

Capture antibodies to the four antigens (NGAL, Cys-C, OPN, and B2mG) were arrayed by the manufacturer onto separate spots on the carbon electrodes of 4-spot 96-well MSD MULTI-SPOT plates. Capture antibodies against IL-18 and KIM-1 were arrayed by the manufacturer onto separate spots on the carbon electrodes of 4-spot 96-well MSD MULTI-SPOT plates, the other two spots were blocked with BSA. MSD supplied the calibrators for the 4-plex assay. Calibrators and biotinylated detection antibodies to IL-18 and KIM-1 were purchased from R\&D Systems (Minneapolis, MN). Urine samples for 4-plex assay were prediluted 1 to 200 with sample dilution buffer (MSD Diluent 2). No dilution of samples was required for the 2-plex assay. Wells were blocked with MSD diluent 2 for $30 \mathrm{~min}$, and $25 \mu \mathrm{L}$ samples and calibrators were added to predestined wells. Plates were incubated for $2 \mathrm{~h}$ at room temperature on a shaker set to $600 \mathrm{rpm}$. Plates were then washed five times with PBS containing 0.05\% Tween-20 (PBS-T) using SkanWasher-300 (Molecular Devices, Sunnyvale, CA).

For the 4-plex assay (NGAL/Cys-C/OPN/B2mG), detection antibodies coupled to a Ruthenium(II)-tris-bipyridine ([Ru(bpy) $\left.{ }_{3}{ }^{2+}\right]$, SULFO-TAG) were blended together and added to wells. For the 2-plex assay (IL-18/KIM1), biotinylated detection antibodies were blended with streptavidin-coupled SULFO-TAG and added to wells. Plates were incubated at room temperature for $2 \mathrm{~h}$ on a shaker $(600 \mathrm{rpm})$. Plates were washed five times with PBS-T on SkanWasher-300 followed by addition of read buffer [buffered Tripropylamine (TPA)].

Detection is based on electrochemical oxidation of $\left[\mathrm{Ru}(\mathrm{bpy})_{3}{ }^{2+}\right]$ in the presence of TPA, an electrochemiluminescence coreactant, leading efficient generation of electrochemiluminescence glow via the high-energy electron transfer reaction between $\mathrm{Ru}(\mathrm{bpy})_{3}{ }^{3+}$ and TPA radical. The electrochemical signal emitted as light was detected in a Sector Imager 2400 with a chargecoupled device, and the signal was analyzed using MSD Workbench and Data Analysis Toolbox v3.0 software. Sample concentrations were back fitted from standard curves generated with a 4-parameter logistic curve fit model with $1 / y^{2}$ weighting. The intra- and interassay precisions were $<3 \%$ and $<5 \%$, respectively, for both 4-plex and 2-plex assays. Standard back-calculated recoveries were $90-110 \%$. Calibrators and sample duplicate correlation variability ranged from $0.12 \%-7.9 \%$.

Statistical analysis. Descriptive statistics were performed to determine differences between infant and maternal characteristics among four GA groups of VLBW infants (Table 1). Shapiro-Wilk test and normal probability plot were used to test for normality of data. Because biomarker values were not normally distributed, Kruskal-Wallis test was used to describe variation in biomarker values based on GA categories. Categorical variables were analyzed using $\chi^{2}$ or Fisher's exact test as appropriate. For all descriptive statistics, a $p<0.05$ was considered statistically significant.

For the regression analysis, biomarkers were converted to natural log to gain normal distribution. For each urine sample obtained, the log of the urine biomarker/creatinine (cr) was calculated. A mixed model analysis, with one random intercept included per child, was performed to explore the impact of the measured variables on the biomarker/creatinine values. GA and AKI were forced into all models. All other maternal and infant demographic variables were explored and removed in a stepwise backward selection model keeping variables with $p<0.2$. The formula ( $\exp ($ beta $)-1) \times 100 \%$ from the regression coefficients was used to express the $\%$ change. SAS 9.2 (SAS Institute Inc., Cary, NC) was used for the all statistical analysis.

\section{RESULTS}

The demographic variability for 123 premature infants categorized by GA (Table 1) shows differences in infant characteristics as expected (birth weight, exposure to indomethacin

Table 1. Demographics by GA categories

\begin{tabular}{|c|c|c|c|c|c|}
\hline & $\leq 26$ wk $(N=52)$ & $26.1-28$ wk $(N=30)$ & $28.1-30$ wk $(N=23)$ & $30.1-36$ wk $(N=18)$ & $p$ \\
\hline \multicolumn{6}{|l|}{ Infant characteristics } \\
\hline Birth weight $(\mathrm{g})$ & $676 \pm 140$ & $948 \pm 184$ & $1118 \pm 219$ & $1224 \pm 245$ & $<0.001$ \\
\hline Small GA & $21(40 \%)$ & $6(20 \%)$ & $6(26 \%)$ & $12(67 \%)$ & $<0.01$ \\
\hline Female & $25(48 \%)$ & $16(53 \%)$ & $7(30 \%)$ & $8(44 \%)$ & 0.39 \\
\hline Race (white) & $35(63 \%)$ & $15(50 \%)$ & $10(42 \%)$ & $7(39 \%)$ & 0.18 \\
\hline Aminoglycoside & $51(98 \%)$ & $30(100 \%)$ & $20(87 \%)$ & $15(83 \%)$ & $<0.01$ \\
\hline Indomethacin & $37(71 \%)$ & $18(60 \%)$ & $4(17 \%)$ & 0 & $<0.001$ \\
\hline Vancomycin & $47(90 \%)$ & $24(80 \%)$ & $10(43 \%)$ & $6(33 \%)$ & $<0.001$ \\
\hline Respiratory support & $50(96 \%)$ & $24(80 \%)$ & $11(48 \%)$ & $4(22 \%)$ & $<0.001$ \\
\hline AKI within $7 \mathrm{~d}$ & $22(42 \%)$ & $2(7 \%)$ & $2(9 \%)$ & $1(6 \%)$ & $<0.001$ \\
\hline 1 min Apgar* & $3 \pm 0.3$ & $4 \pm 0.5$ & $5 \pm 0.5$ & $5 \pm 0.6$ & $<0.001$ \\
\hline 5 min Apgar* & $6 \pm 0.3$ & $6 \pm 0.3$ & $8 \pm 0.3$ & $7 \pm 0.3$ & $<0.001$ \\
\hline \multicolumn{6}{|l|}{ Maternal characteristics } \\
\hline Hypertension & $12(23 \%)$ & $14(47 \%)$ & $9(39 \%)$ & $13(72 \%)$ & $<0.001$ \\
\hline Antenatal steroids & $42(81 \%)$ & $21(70 \%)$ & $20(87 \%)$ & $13(72 \%)$ & 0.77 \\
\hline Indomethacin & $3(6.0 \%)$ & $1(3 \%)$ & 0 & 0 & 0.13 \\
\hline Preeclampsia & $6(11.5 \%)$ & $12(40 \%)$ & $6(26 \%)$ & $9(50 \%)$ & $<0.002$ \\
\hline
\end{tabular}

\footnotetext{
* Apgar mean $\pm \mathrm{SE}$.
} 
Table 2. Variation of candidate urine AKI biomarker levels by GA

\begin{tabular}{|c|c|c|c|c|c|}
\hline & $\leq 26 \mathrm{wk}(N=52)$ & $26.1-28$ wk $(N=30)$ & $28.1-30 \mathrm{wk}(N=23)$ & $30.1-36$ wk $(N=18)$ & $p$ \\
\hline NGAL $(\mathrm{ng} / \mathrm{mL})$ & $351(271-456)$ & $231(161-333)$ & $145(96-218)$ & $85(53-134)$ & $<0.001$ \\
\hline IL-18 (pg/mL) & $42(2.0-67)$ & $41(21-81)$ & $30(14-63)$ & $67(29-155)$ & 0.57 \\
\hline KIM-1 (pg/mL) & $226(184-277)$ & $158(117-212)$ & $155(112-213)$ & $143(99,207)$ & 0.04 \\
\hline Cys-C (ng/mL) & $911(570-1454)$ & $457(195-1069)$ & $230(87-608)$ & $133(27-657)$ & 0.01 \\
\hline OPN (ng/mL) & $177(142-221)$ & $121(81-181)$ & $145(92-229)$ & $83.5(40-177)$ & 0.13 \\
\hline $\mathrm{B} 2 \mathrm{mG}(\mathrm{mg} / \mathrm{mL})$ & $0.8(0.7-1.0)$ & $1.0(0.7-1.4)$ & $0.9(0.6-1.3)$ & $0.3(0.1-0.5)$ & $<0.01$ \\
\hline Total samples & 215 & 102 & 80 & 67 & \\
\hline Samples per infant, Median (range) & $4(1-7)$ & $3(1-7)$ & $3(1-6)$ & $3(1-6)$ & \\
\hline
\end{tabular}

Geometric mean $(95 \% \mathrm{CI})$ for all urine measurements.

$N$, number of subjects.

Table 3. Variation of candidate urine AKI biomarker levels corrected for urine creatinine by GA

\begin{tabular}{|c|c|c|c|c|c|}
\hline & $\leq 26$ wk $(N=52)$ & $26.1-28$ wk $(N=30)$ & $28.1-30 \mathrm{wk}(N=23)$ & $30.1-36$ wk $(N=18)$ & $p$ \\
\hline NGAL/cr & $2713(1888-3898)$ & $1355(814-2253)$ & $546.5(310-965)$ & $447.8(235-853)$ & $<0.001$ \\
\hline IL-18/cr & $329(186-582)$ & $252(113-561)$ & $112(46-273)$ & $357(129-983)$ & 0.21 \\
\hline $\mathrm{KIM}-1 / \mathrm{cr}$ & $1802(1378-2357)$ & $918(617-1366)$ & $637(416-976)$ & $803(493-1310)$ & $<0.001$ \\
\hline Cys-C/cr & $5790(3083-10877)$ & $2224(706-7004)$ & $273(72-1032)$ & $140(15-1275)$ & $<0.001$ \\
\hline $\mathrm{B} 2 \mathrm{mG} / \mathrm{cr}$ & $5.3(3.4-8.4)$ & $4.4(1.9-10.3)$ & $1.1(0.4-3.0)$ & $0.4(0.1-2.2)$ & $<0.001$ \\
\hline $\mathrm{OPN} / \mathrm{cr}$ & $1152(715-1856)$ & $550(230-1311)$ & $174(64-480)$ & $123(23-662)$ & $<0.001$ \\
\hline Creatinine $(\mathrm{cr}), \mathrm{mg} / \mathrm{mL}$ & $0.09 \pm 0.06$ & $0.11 \pm 0.10$ & $0.14 \pm 0.06$ & $0.15 \pm 0.03$ & $<0.001$ \\
\hline
\end{tabular}

Geometric mean $(95 \% \mathrm{CI})$ for all urine measurements.

$N$, number of subjects.

and aminoglycosides, respiratory support, Apgar scores at 1 and $5 \mathrm{~min}$, AKI, and small for GA; all $p<0.01)$. Maternal preeclampsia $(p<0.02)$ and hypertension $(p<0.001)$ were different among groups $(p<0.01)$.

As GA increased, urine NGAL, KIM-1, Cys-C, and B2mG values progressively decreased, but no significant differences were seen for other biomarkers (Table 2). When corrected for urine $\mathrm{cr}$, all biomarker/cr ratios progressively declined with GA except for IL-18/cr (Table 3).

After controlling for other potential confounders, GA independently impacted NGAL/cr, OPN/cr, and B2MG/cr but not KIM-1/cr, IL-18/ cr, or Cys-C/cr. Birth weight was independently associated with Cys-C/cr. The use of blood pressure support medication and indomethacin was independently associated with Kim-1/cr levels. Female gender was independently associated with higher NGAL/cr and IL-18/cr and lower Kim-1/cr. Vancomycin was independently associated with OPN/cr values. Race was independently associated with $\mathrm{OPN} / \mathrm{cr}$ and $\mathrm{B} 2 \mathrm{mG} / \mathrm{cr}$ values (Table 4).

\section{DISCUSSION}

Baseline values of urine NGAL, KIM-1, Cys-C, and B2mG decrease with increasing GA. With correction to urine cr, these markers and OPN decreased with increasing GA. IL-18 (with or without correction for urine creatinine) did not differ across GA categories. Controlling for other potential clinical and demographic confounders with regression analysis shows that NGAL/cr, OPN/cr, and B2mG/cr are independently associated with GA.

Even though this study was not designed to determine whether these biomarkers predict AKI, we forced AKI into the models to determine whether variables such as GA were simply a common variable to explain high biomarker values and AKI. We found that GA continued to be associated with higher biomarker values, even when controlling for AKI, which suggests that these biomarkers are affected by GA and not simply that infants with higher GA have more AKI.

Our conclusions and values for the differences in urine NGAL values based on birth weight (data not shown) were similar to those documented by Lavery et al. (27). Besides confirming previous results on baseline values of urine NGAL in VLBW infants, our study provides baseline values and evidence that these biomarkers are affected by GA in other urine biomarkers (KIM-1, Cys-C, B2-MG, and OPN) but not IL-18. These normative data will help investigators design and analyze AKI biomarker studies.

In 2008, Huynh et al. (29) described urine NGAL levels of infants (birth weight $>750 \mathrm{~g}$ ) and showed differences between males and females but did not stratify them according to GA or birth weight categories. Interestingly, the baseline urine NGAL values in our cohort were similar to values found by Huynh et al. (29) for those whose birth weight is $>750 \mathrm{~g}$, but the overall values in our cohort were higher likely because our population included many infants with birth weight $<750 \mathrm{~g}$. Also, our sample showed higher variability among NGAL levels likely due to differences in our sample population (Huynh excluded those with clinical risk factors for AKI, whereas we included a more heterogeneous premature infant population). Our data show an independent association between urine biomarker values and female gender which confirms the findings by Huynh et al. which showed consistently higher urine NGAL values in premature females.

Compared with other critically ill pediatric patients who were on ventilator support and required ionotropic medications $(14,30)$, baseline NGAL values are significantly higher in our population while urine IL-18 levels were similar, likely because their cohort was made up of children with multiorgan failure. Similarly baseline values of urine NGAL (but not 
Table 4. Mixed model regression to predict log of urine biomarker/creatinine

\begin{tabular}{|c|c|c|c|}
\hline Parameter & $\begin{array}{c}\text { Point estimate } \\
\% \text { change }\end{array}$ & $\begin{array}{l}95 \% \text { CI for } \\
\% \text { change }\end{array}$ & $p$ \\
\hline \multicolumn{4}{|l|}{ NGAL/cr* } \\
\hline GA & $-23.1 \%$ per wk & $-32.8,-13.3$ & $<0.001$ \\
\hline Female & $62.7 \%$ & $104.9 \%, 20.4 \%$ & 0.003 \\
\hline Race (white) & $-39.8 \%$ & $-81.7 \%, 2.1 \%$ & 0.06 \\
\hline AKI present & $39.6 \%$ & $-10.0 \%, 89.1 \%$ & 0.11 \\
\hline Infant UAC & $43.1 \%$ & $-5.8 \%, 92.1 \%$ & 0.08 \\
\hline \multicolumn{4}{|l|}{ KIM-1/cr† } \\
\hline GA & $-5.94 \%$ per wk & $-13.61 \%, 1.717 \%$ & 0.12 \\
\hline Female & $-39.85 \%$ & $-7.474 \%,-72.23 \%$ & 0.01 \\
\hline Race (white) & $-27.01 \%$ & $-60.23 \%, 6.213 \%$ & 0.10 \\
\hline AKI & $-3.5 \%$ & $-45.14 \%, 38.22 \%$ & 0.87 \\
\hline Infant indomethacin & $40.2 \%$ & $3.342 \%, 76.98 \%$ & 0.03 \\
\hline $\begin{array}{c}\text { Medication for } \\
\text { hypotension }\end{array}$ & $59.8 \%$ & $16.93 \%, 102.71$ & 0.006 \\
\hline \multicolumn{4}{|l|}{ IL-18/cr $\ddagger$} \\
\hline GA & $-0.8 \%$ per wk & $-16.2 \%, 14.6 \%$ & 0.91 \\
\hline Female gender & $92.2 \%$ & $16.9 \%, 167.6 \%$ & 0.01 \\
\hline AKI & $84.7 \%$ & $-2.8 \%, 172.1 \%$ & 0.06 \\
\hline \multicolumn{4}{|l|}{ Cys-C/cr§ } \\
\hline GA & $-20.8 \%$ per wk & $-54.1 \%, 12.6 \%$ & 0.22 \\
\hline Birth weight & $-37.8 \%$ per $100 \mathrm{~g}$ & $-69.1 \%,-6.5$ & 0.02 \\
\hline Female gender & $51.8 \%$ & $-40.6 \%, 144.2 \%$ & 0.26 \\
\hline AKI & $-34.5 \%$ & $-59.0,40.3 \%$ & 0.49 \\
\hline \multicolumn{4}{|l|}{$\mathrm{OPN} / \mathrm{cr} \|$} \\
\hline GA & $-34.8 \%$ per wk & $-50.6 \%,-19.0 \%$ & $<.0001$ \\
\hline AKI & $-20.1 \%$ & $-93.4 \%, 53.2 \%$ & 0.58 \\
\hline Infant aminoglycoside & $-222.8 \%$ & $-492.6 \%, 47.0 \%$ & 0.10 \\
\hline Infant vancomycin & $89.1 \%$ & $2.3 \%, 175.9 \%$ & 0.04 \\
\hline Race (white) & $-77.2 \%$ & $-142.1 \%,-12.3 \%$ & 0.02 \\
\hline \multicolumn{4}{|l|}{$\mathrm{B} 2 \mathrm{mG} / \mathrm{cr} \|$} \\
\hline GA & $-30.6 \%$ per wk & $-43.0 \%,-18.1 \%$ & $<.0001$ \\
\hline Female gender & $23.2 \%$ & $-30.9 \%, 77.3 \%$ & 0.39 \\
\hline Race (white) & $-69.1 \%$ & $-123.3 \%,-15.0 \%$ & 0.01 \\
\hline AKI present & $-13.7 \%$ & $-72.8 \%, 45.4 \%$ & 0.64 \\
\hline
\end{tabular}

Mixed linear model with random intercept per subject for log biomarker/ creatinine. The formula $(\exp ($ beta $)-1) \times 100 \%$ from the regression coefficients was used to express the $\%$ change. GA and AKI forced into all models, others selected for $p<0.2$.

$* \log (\mathrm{NGAL} / \mathrm{cr})=\mathrm{GA}+$ gender $+\mathrm{AKI}+$ infant UAC (umbilical arterial catheter + race.

$\dagger \log (\mathrm{KIM}-1 / \mathrm{cr})=\mathrm{GA}+$ gender $+\mathrm{AKI}+$ infant indomethacin + med for hypotension + race.

\$ $\log (\mathrm{IL}-18 / \mathrm{cr})=\mathrm{GA}+$ gender $+\mathrm{AKI}$.

$\S \log ($ Cys-C/cr $)=\mathrm{GA}+$ birth weight + gender + AKI.

$\| \log (\mathrm{OPN} / \mathrm{cr})=\mathrm{GA}+$ race + infant aminoglycoside + infant vancomycin. II $\log (\mathrm{B} 2 \mathrm{mG} / \mathrm{cr})=\mathrm{GA}+$ gender $+\mathrm{AKI}+$ race.

IL-18, B2MG, or OPN) were higher in a cohort of children evaluated in a pediatric emergency department (31).

The strength of this study includes the heterogeneous group of VLBW infants and the evaluation of six candidate biomarkers. However, we acknowledge several limitations. Even though we report the number of infants with known AKI in each category, we acknowledge that some infants may have sustained AKI but were "missed" if SCr levels around the time of insult were not performed; thus, from our study, the impact of AKI on these biomarkers cannot be ascertained. Although we controlled for AKI in our regression model, it is possible that the reason higher levels were seen in those with lower GA is due to higher incidence of AKI. Second, we acknowledge that there could be other variables which we did not account for that could explain variations in biomarker levels. Therefore, inferences from this analysis should be taken in context of the above limitations. Future studies to determine the ability of these and other biomarkers to detect AKI and mortality are greatly needed.

In conclusion, the normative urine values of NGAL, KIM-1, OPN, Cys-C, and B2mG (but not IL-18) are higher in the most premature. One must acknowledge these differences when designing validation studies to find candidate biomarkers of AKI. Whether clinicians and researchers will need to adjust for GA, birth weight, and gender differences when using these biomarkers is yet to be determined.

Acknowledgments. We thank Amy Logue, RN, BSN, Faisal Schwab, Shamima Akhter, Amandi Liwo, and Sonja Jordan for their assistance in data collection and management of the study. We thank all of the nurses in the NICU for their diligence in collecting samples.

\section{REFERENCES}

1. Eichenwald EC, Stark AR 2008 Management and outcomes of very low birth weight. N Engl J Med 358:1700-1711

2. Askenazi DJ, Griffin R, McGwin G, Carlo W, Ambalavanan N 2009 Acute kidney injury is independently associated with mortality in very low birthweight infants: a matched case-control analysis. Pediatr Nephrol 24:991-997

3. Askenazi DJ, Ambalavanan N, Goldstein SL 2009 Acute kidney injury in critically ill newborns: what do we know? What do we need to learn? Pediatr Nephrol 24:265-274

4. Koralkar R, Ambalavanan N, Levitan EB, McGwin G, Goldstein S, Askenazi D 2011 Acute kidney injury reduces survival in very low birth weight infant. Pediatr Res 69:354-358

5. Bennett M, Dent CL, Ma Q, Dastrala S, Grenier F, Workman R, Syed H, Ali S, Barasch J, Devarajan P 2008 Urine NGAL predicts severity of acute kidney injury after cardiac surgery: a prospective study. Clin J Am Soc Nephrol 3:665-673

6. Coca SG, Yalavarthy R, Concato J, Parikh CR 2008 Biomarkers for the diagnosis and risk stratification of acute kidney injury: a systematic review. Kidney Int 73:1008-1016

7. Dent CL, Ma Q, Dastrala S, Bennett M, Mitsnefes MM, Barasch J, Devarajan P 2007 Plasma neutrophil gelatinase-associated lipocalin predicts acute kidney injury, morbidity and mortality after pediatric cardiac surgery: a prospective uncontrolled cohort study. Crit Care 11:R127

8. Diamanti-Kandarakis E, Livadas S, Kandarakis SA, Margeli A, Papassotiriou I 2008 Serum concentrations of atherogenic proteins neutrophil gelatinase-associated lipocalin and its complex with matrix metalloproteinase- 9 are significantly lower in women with polycystic ovary syndrome: hint of a protective mechanism? Eur J Endocrinol 158:525-531

9. Herrero-Morín JD, Málaga S, Fernández N, Rey C, Diéguez MA, Solís G, Concha A, Medina 2007 A Cystatin C and beta2-microglobulin: markers of glomerular filtration in critically ill children. Crit Care 11:R59

10. Liangos O, Tighiouart H, Perianayagam MC, Kolyada A, Han WK, Wald R, Bonventre JV, Jaber BL 2009 Comparative analysis of urinary biomarkers for early detection of acute kidney injury following cardiopulmonary bypass. Biomarkers $14: 423-431$

11. Ling W, Zhaohui N, Ben H, Leyi G, Jianping L, Huili D, Jiaqi Q 2008 Urinary IL-18 and NGAL as early predictive biomarkers in contrast-induced nephropathy after coronary angiography. Nephron Clin Pract 108:c176-c181

12. Mishra J, Ma Q, Kelly C, Mitsnefes M, Mori K, Barasch J, Devarajan P 2006 Kidney NGAL is a novel early marker of acute injury following transplantation. Pediatr Nephrol 21:856-863

13. Parikh CR, Abraham E, Ancukiewicz M, Edelstein CL 2005 Urine IL-18 is an early diagnostic marker for acute kidney injury and predicts mortality in the intensive care unit. J Am Soc Nephrol 16:3046-3052

14. Zappitelli M, Washburn KK, Arikan AA, Loftis L, Ma Q, Devarajan P, Parikh CR, Goldstein SL 2007 Urine neutrophil gelatinase-associated lipocalin is an early marker of acute kidney injury in critically ill children: a prospective cohort study. Crit Care 11:R84

15. Mishra J, Dent C, Tarabishi R, Mitsnefes MM, Ma Q, Kelly C, Ruff SM, Zahedi K, Shao M, Bean J, Mori K, Barasch J, Devarajan P 2005 Neutrophil gelatinaseassociated lipocalin (NGAL) as a biomarker for acute renal injury after cardiac surgery. Lancet 365:1231-1238

16. Parikh CR, Mishra J, Thiessen-Philbrook H, Dursun B, Ma Q, Kelly C, Dent C, Devarajan P, Edelstein CL 2006 Urinary IL-18 is an early predictive biomarker of acute kidney injury after cardiac surgery. Kidney Int 70:199-203

17. Goldstein SL 2007 Kidney function assessment in the critically ill child: is it time to leave creatinine behind? Crit Care 11:141 
18. Parikh CR, Devarajan P 2008 New biomarkers of acute kidney injury. Crit Care Med 36:S159-S165

19. Devarajan P 2008 Neutrophil gelatinase-associated lipocalin (NGAL): a new marker of kidney disease. Scand J Clin Lab Invest Suppl 241:89-94

20. Mishra J, Ma Q, Prada A, Mitsnefes M, Zahedi K, Yang J, Barasch J, Devarajan P 2003 Identification of neutrophil gelatinase-associated lipocalin as a novel early urinary biomarker for ischemic renal injury. J Am Soc Nephrol 14:2534-2543

21. Han WK, Bailly V, Abichandani R, Thadhani R, Bonventre JV 2002 Kidney Injury Molecule-1 (KIM-1): a novel biomarker for human renal proximal tubule injury. Kidney Int 62:237-244

22. Liangos O, Perianayagam MC, Vaidya VS, Han WK, Wald R, Tighiouart H, MacKinnon RW, Li L, Balakrishnan VS, Pereira BJ, Bonventre JV, Jaber BL 2007 Urinary $N$-acetyl-beta-(D)-glucosaminidase activity and kidney injury molecule-1 level are associated with adverse outcomes in acute renal failure. J Am Soc Nephrol 18:904-912

23. Trof RJ, Di Maggio F, Leemreis J, Groeneveld AB 2006 Biomarkers of acute renal injury and renal failure. Shock 26:245-253

24. Devarajan P 2007 Emerging biomarkers of acute kidney injury. Contrib Nephrol 156:203-212

25. Parikh CR, Edelstein CL, Devarajan P, Cantley L 2007 Biomarkers of acute kidney injury: early diagnosis, pathogenesis, and recovery. J Investig Med 55:333-340
26. Vaidya VS, Ford GM, Waikar SS, Wang Y, Clement MB, Ramirez V, Glaab WE, Troth SP, Sistare FD, Prozialeck WC, Edwards JR, Bobadilla NA, Mefferd SC, Bonventre JV 2009 A rapid urine test for early detection of kidney injury. Kidney Int 76:108-114

27. Lavery AP, Meinzen-Derr JK, Anderson E, Ma Q, Bennett MR, Devarajan P, Schibler KR 2008 Urinary NGAL in premature infants. Pediatr Res 64:423-428

28. Mehta RL, Kellum JA, Shah SV, Molitoris BA, Ronco C, Warnock DG, Levin A; Acute Kidney Injury Network 2007 Acute Kidney Injury Network: report of an initiative to improve outcomes in acute kidney injury. Crit Care 11:R31

29. Huynh TK, Bateman DA, Parravicini E, Lorenz JM, Nemerofsky SL, Sise ME, Bowman TM, Polesana E, Barasch JM 2009 Reference values of urinary neutrophil gelatinase-associated lipocalin in very low birth weight infants. Pediatr Res 66:528532

30. Washburn KK, Zappitelli M, Arikan AA, Loftis L, Yalavarthy R, Parikh CR, Edelstein CL, Goldstein SL 2008 Urinary interleukin-18 is an acute kidney injury biomarker in critically ill children. Nephrol Dial Transplant 23:566-572

31. Du Y, Zappitelli M, Mian A, Bennett M, Ma Q, Devarajan P, Mehta R, Goldstein SL 2011 Urinary biomarkers to detect acute kidney injury in the pediatric emergency center. Pediatr Nephrol 26:267-274 